\title{
Report on Optical Absorption, Steady-state Emission and Time-resolved Emission Spectroscopy of Carbazole-based Conjugated Polymers
}

\author{
R. K. Dey ${ }^{a}$, A. M. H. Chowdhury ${ }^{\mathrm{b}}$, N. Chawdhury ${ }^{\mathrm{b}}$, and A. P. Monkman ${ }^{\mathrm{c}}$ \\ ${ }^{a}$ Department of Physics and Astronomy, The University of Western Ontario, London, Ontario, \\ Canada \\ ${ }^{\mathrm{b}}$ Department of Physics, Shahjalal University of Science \& Technology, Sylhet-3114, Bangladesh \\ ${ }^{\mathrm{c}}$ Department of Physics, University of Durham, Durham DHI 3LE, England \\ Received 22 March 2010, accepted in final revised form 7 December 2010

\begin{abstract}
Opto-electronic properties of three new carbazole based co-polymers were investigated. The decay kinetics of triplet emission of the co-polymers was observed. Several findings such as optical band-gap, singlet to singlet transition energies, triplet to singlet transition energies, exchange energies $\left(\Delta E_{\mathrm{ST}}\right)$ have been presented. In comparison to the other material carbazole-oxadiazole based polymer with OMe, P1, $\mathrm{H}_{21} \mathrm{C}_{10}, \mathrm{P} 2$ and $\mathrm{H}_{12} \mathrm{C}_{10} \mathrm{O}$, P3 exchange energy is below $0.5 \mathrm{eV}$ and triplet energy is significantly higher. The energy gaps between singlet and triplet excited states are $0.44-0.47 \mathrm{eV}$. From decay kinetics the triplet excited state life-times were found to be several orders of ms at 10-13 K that reduces to less than 3 $\mathrm{ms}$ at room temperature.
\end{abstract}

Keywords: Time resolved emission spectroscopy; Carbazole; Conjugated polymers.

(c) 2011 JSR Publications. ISSN: 2070-0237 (Print); 2070-0245 (Online). All rights reserved. doi:10.3329/jsr.v3i1.4666

J. Sci. Res. 3 (1), 13-24 (2011)

\section{Introduction}

Conjugated polymers have attracted considerable research interest during the past decade due to their great potential in applications such as electrically pumped lasers and solar cells, but mainly as active materials for displays device [1]. Recently, research in this field has revealed that the dependence of HOMO- LUMO energy level on carbazole moiety and electron deficient comonomer, respectively though the solar cell performance does not depend upon the energy level of the material but on the overall organization of the polymer [1]. The latter, being based on polymer light-emitting diodes PLED, transistors,

\footnotetext{
*Corresponding author: rdey3@uwo.ca
} 
and photovoltaic cells, Blouin et al. [2] described the device characteristics as structureproperties relationship. Mori and Kijima [3] have reported the electroluminescence properties of carbazole-containing 2,6-naphthalene-based conjugated polymers where the device fabricated with the random copolymer exhibited highest performances showing a maximum brightness of $8370 \mathrm{~cd} / \mathrm{m} 2$ at $13 \mathrm{~V}$ and a maximum efficiency of $2.16 \mathrm{~cd} / \mathrm{A}$ at 7 $\mathrm{V}$ [3]. Generally two types of excited species are formed in working PLED's potentially emissive singlet states and non-emissive triplet states. Rothe et al. [4] showed that the triplet transfer process investigated did not obey simple exponential transfer rates. Instead, the observed results are more complex and always dispersive i.e., vibronic. Theoretically the branching ratio (the branching fraction for decay is the fraction of particles which decay by an individual decay mode with respect to the total number of particles which decay) favors the triplet state 3:1 due to the statistical nature of the recombination process of the carriers. However, spin-dependent recombination suggests a ratio closer to $1: 1$, depending on the individual polymer. The implications of this clearly show that triplet states have equal importance compared to the singlet state. However, there is much more known about the fluorescent singlet exciton. This fact is understandable bearing in mind that the triplet is, due to spin conservation, highly non-emissive, which impedes its observation with almost every direct luminescence measurement. For experimental studies the triplet manifold is usually populated via photo-excitation followed by intersystem crossing, which is more or less efficient depending on the conjugated polymer. However, the problem of non-emissive triplet states (forbidden region) has been circumvented by investigating a system of carbazole based polymers.

Carbazole polymers have been investigated as photoconductors and hole-transporting materials [5]. Yi et. al. [6] reported the preparation of a 2,7-linked carbazole polymer with triarylamine functional groups attached at the 9-position of carbazole repeat units that results as blue-emitting materials. Recently novel conjugated carbazole polymers which was based on the alkyne-linked 1, 8-carbazole were synthesized by the Sonogashira cross-coupling reaction and acetylenic oxidative coupling reaction [7].

The inclusion of carbazole induces strong spin-orbit coupling so that triplet state emission can be detected using conventional spectroscopy techniques [8, 9]. Molecular structures of the carbazole-based polymers were observed where solvent-induced aggregation of 2, 7 carbazole-based conjugated polymers include their capability to emit in the blue region of the visible spectrum [10]. The decay curves, life-time of the triplet excited state, fluorescence and phosphorescence spectra are presented and compared.

\section{Experimental Section}

A series of carbazole based polymers was investigated using optical spectroscopy and the chemical structures of those polymers are shown in the following figure. For simplicity, we indicate those as P1, P2 and P3 in this study. Fig. 1 shows the chemical structures of three polymers that are used in this study. 

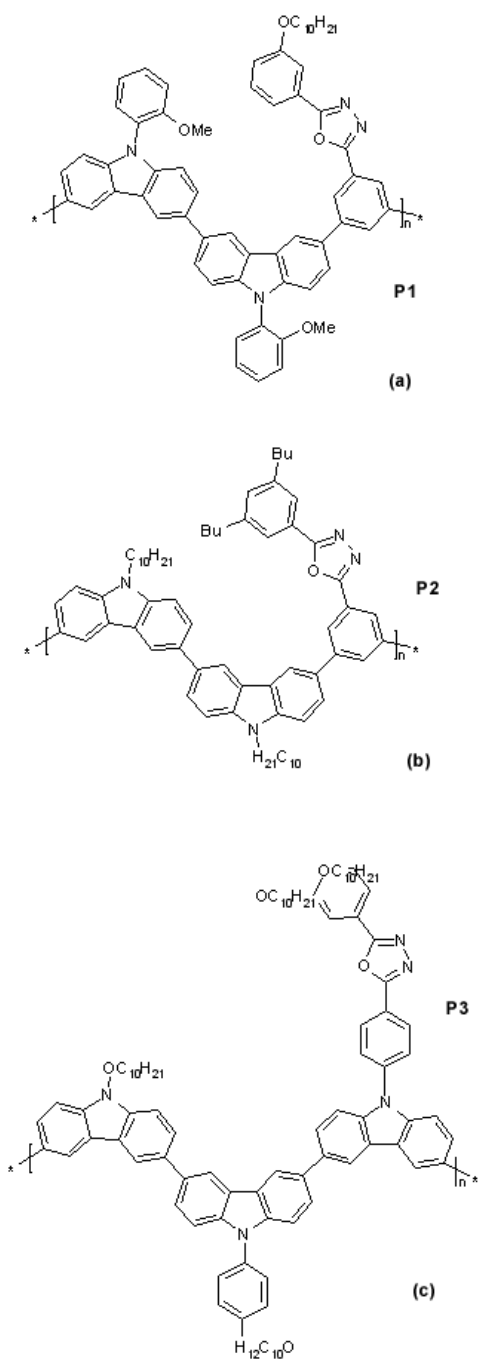

Fig. 1. Chemical structures of three polymers that used in this study. (a) Carbazole-oxadiazole based polymer with OMe, P1; (b) Carbazole based polymer with $\mathrm{H}_{21} \mathrm{C}_{10}$, P2; (c) Carbazole-oxadiazole based polymer with $\mathrm{H}_{12} \mathrm{C}_{10} \mathrm{O}$, P3.

P1, P2 and P3 polymers used in the work obtained as dry powder directly after synthesis. The materials were kept in dark and in glovebox before being dissolved. The solutions of concentrations $0.02 \mathrm{mg} / \mu \mathrm{l}$ were prepared using clorobenzene as solvent. For the optical measurements described here, the thin solid film samples were prepared by spin coating with $2000 \mathrm{rpm}$ on to $1 \mathrm{~mm}$ thick 'Spectrosil B' quartz discs. Optical absorption of films and solutions were measured at room temperature using a commercial 
Perkin-Elmer spectrophotometer. The fluorescence emission spectra of the solution and solid samples were measured using a Fluoromax spectrometer. The excitation lines were chosen such that the absorption was enough to excite the complex.

For the time-resolved spectroscopy studies a pulsed Nd: yttrium aluminum garnet laser pulse width 120 ps; maximum pulse energy at the excitation wavelength $355 \mathrm{~nm}, 7 \mathrm{~mJ}$; repetition rate 1-10 $\mathrm{Hz}$ was used to excite the singlet manifold of polymers. The light emitted by the sample was monochromated and subsequently detected by the gated intensified charge coupled device (CCD camera 4 picos, Stanford Computer Optics). "Gated" refers to an adjustable time delay after the trigger pulse provided by a fast photodiode responding to the excitation laser set before the start of detection, with the duration of detection also being tunable, the time resolution is limited by a minimum gate width of 200 ps. These spectra are characterized by two tuneable time periods: the time after pulsed excitation until the start of the light detection (delay time) and the duration of the light detection (integration time). In order to smooth laser intensity fluctuations, every spectrum was obtained by accumulating up to 50 laser shots. A temperature controlled displex helium cryostat allowed us to maintain any sample temperature between 300 and $11 \mathrm{~K}$. We used Monochromator (TRIAX 180) in the experiments of emission spectroscopy as detector.

\section{Results and Discussion}

\section{The optical absorption and steady-state emission spectra}

The optical absorption spectra, and steady-state emission spectra of P1, P2 and P3 polymers in terms of energies are shown in Figs. 2 (a, b).

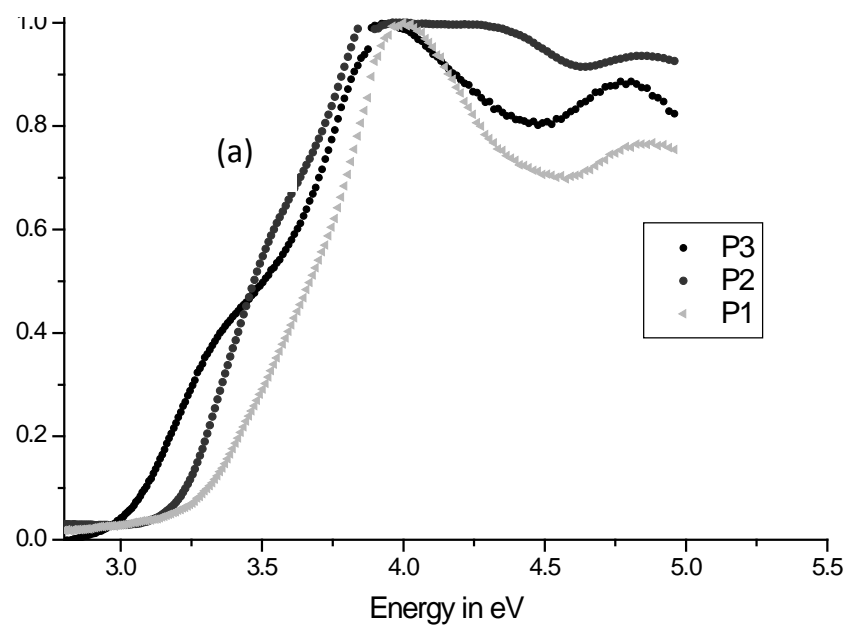




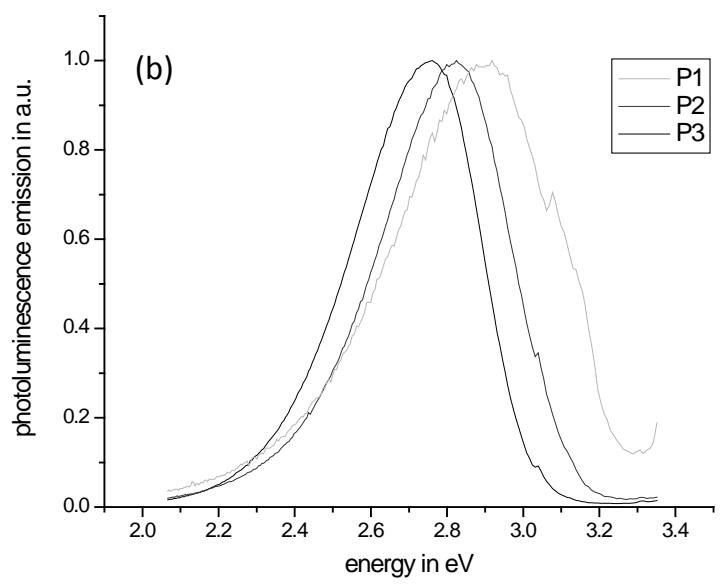

Fig. 2. (a) Optical absorption spectra, (b) steady-state emission spectra of P1, P2 and P3 polymers in terms of energies.

The emission peaks are at $2.92 \mathrm{eV}, 2.82 \mathrm{eV}$ and $2.76 \mathrm{eV}$ for P1, P2 and P3, respectively. The onset of optical absorption indicates the band-gaps of these materials. Here, the onset of absorption is $3.25 \mathrm{eV}, 3.20 \mathrm{eV}$ and $3.10 \mathrm{eV}$ for P1, P2 and P3 polymers respectively. The energies of the singlet and the triplet peaks decrease along with the optical gap, and a constant singlet-triplet energy gap of $0.7 \mathrm{eV} \pm 0.1 \mathrm{eV}$ is seen for the polymers [11, 12]. The absorption peaks of P1, P2 and P3 are at $4.0 \mathrm{eV}, 3.90 \mathrm{eV}$ and 3.87 $\mathrm{eV}$. Here $\Delta \mathrm{E}$ between absorption and emission peak is $1.08 \mathrm{eV}, 1.08 \mathrm{eV}$ and $1.11 \mathrm{eV}$ respectively. These values are very much higher than common conjugated polymers such as PPV (poly (p-phenylene vinylene)), MEH-PPV (2-methoxy-5 [2'-ethyl-hexyl)axy]) etc. Therefore, we attribute this band as due to the absorption into second singlet excited state $S_{2}$ from $S_{0}$, i.e. $S_{0} \rightarrow S_{2}$. However, we observe weak shoulders at lower energy side at $3.40 \mathrm{eV}, 3.25 \mathrm{eV}$ and $3.30 \mathrm{eV}$ for P1, P2 and P3 polymers respectively. Considering these absorption bands, we get stoke's shift of $0.48 \mathrm{eV}, 0.43 \mathrm{eV}$ and $0.54 \mathrm{eV}$ respectively. Therefore, I attribute these shoulders as due to absorption into first excited state $\mathrm{S}_{1}$ from $\mathrm{S}_{0}$, i.e. $\mathrm{S}_{0} \rightarrow \mathrm{S}_{1}$.

In PPV (poly (p-phenylene vinylene)), the peak of the emission spectrum is near 2.2 $\mathrm{eV}$ [13] and the peak of the excitation spectrum is at $2.4 \mathrm{eV}$ [14] which gives rise to stoke's shift of $0.2 \mathrm{eV}$. Stoke's shift decreases with increasing morphological order in the samples. In the case of 2-methoxy-5 [2'-ethyl-hexyl)axy] derivative of PPV, MEH-PPV, the stokes shift is measured to be $0.2-0.3 \mathrm{eV}$ in typical samples[15] , and in aligned and oriented sample. It is less than $0.1 \mathrm{ev}$ (0-0 peak in absorption red-shifts from $2.25 \mathrm{eV}$ to $2.13 \mathrm{eV})[16]$. 
In comparing absorption energies of those polymers, as shown Fig. 2 (a), we get for $\mathrm{S}_{0}$ $\rightarrow \mathrm{S}_{1}$, in absorption, $\mathrm{P} 1(3.40 \mathrm{eV})>\mathrm{P} 3(3.30 \mathrm{eV})>\mathrm{P} 2(3.25 \mathrm{eV})$. P2 polymer contains a carbazole with an alkyl group. However, the other two polymers contain carbazoleoxadiazole systems which have aryl groups. We see that $\mathrm{P} 2$ has lowest energy in its peak. This is because; an alkyl group of P2 polymer activates the carbazole ring system by increasing the electron density and makes the compound easier to oxidize, then the corresponding aryl-substitutes compound. Therefore, alkyl group contributes to the delocalization in the carbazole system. As the aryl group is twisted with respect to the carbazole ring system, it is not involved in delocalized of $\pi$-electrons in the carbazole ring system.

\section{Time resolved emission spectroscopy}

Time resolved photoluminescence spectra are characterized by two tunable time periods: the time after pulsed excitation until the start of the light detection (delay time) and the duration of the light detection (integration time). Typically, 50 laser shots are accumulated for a single spectrum. In order to obtain the decay kinetics, each spectrum was integrated over the desired energy region and plotted versus the appropriate time. The overall sub nanosecond time resolution of the setup is accompanied by a huge dynamic range.

Generally, when attaching electron withdrawing substituents (such as bromine) to conjugated molecules, the electron density in the $\pi$-system of the conjugated molecule is decreased. Consequently, the molecule is stabilized and the oxidation potential is increased, corresponding to a shift of the HOMO level to lower energy [17] Apparently, for carbazole such a stabilizing effect is stronger for substitution at the 3 and 6 positions (see Fig. 3) than for substitution at the 2 and 7 positions. This can be explained by the fact that for the HOMO of the carbazole molecule, the electronic density at the 2 and 7 positions is less than at the $3 \mathrm{HOMO}$ level of carbazole compounds by substitution at the 3 , and 6 positions. Therefore, substituting the 2 and 7 positions with electron withdrawing groups have less influence on the electron density of the carbazole molecule than substituting the 3 and 6 positions [18].

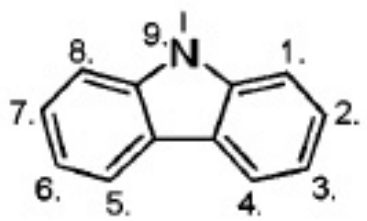

Fig. 3. Different positions of an ordinary polymer.

The materials P1, P2 and P3 studied in this thesis are conjugated polymers containing carbazole unit. The conjugated main-chain is attached to 3 and 6 positions for the 
stabilization of the polymer and increased delocalization of electrons through polymer chain.

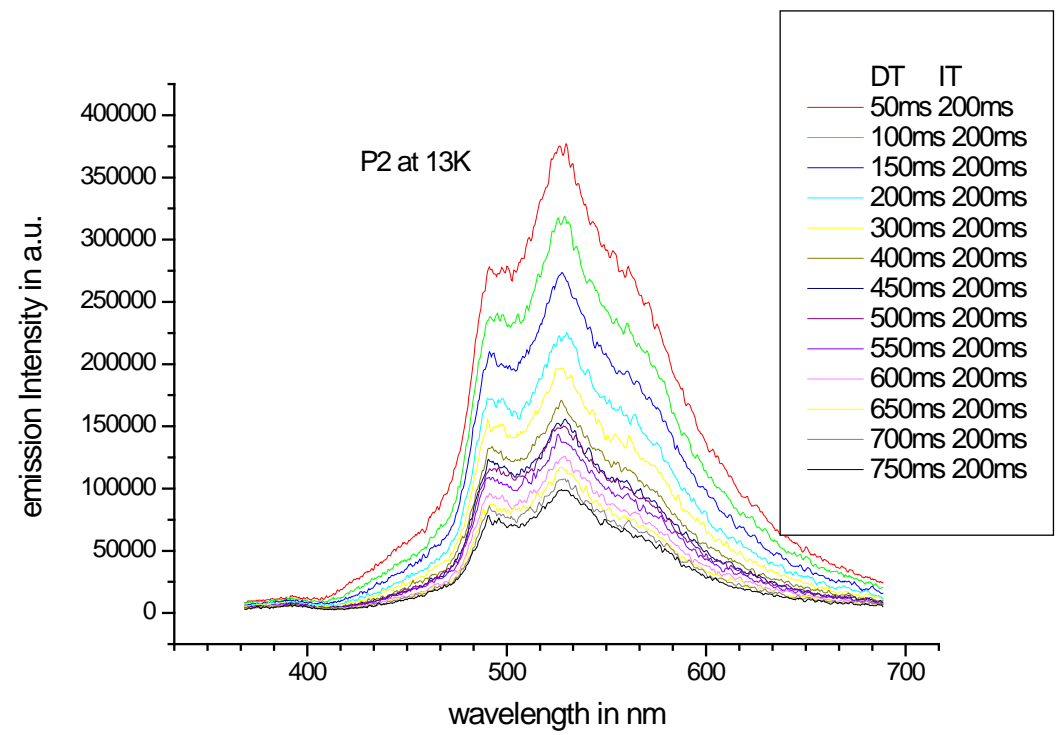

Fig. 4. Time resolved phosphorescence spectra for with a particular integration time (IT) and different delay time (DT) at low temperature $(10 \mathrm{~K})$ and at RT.

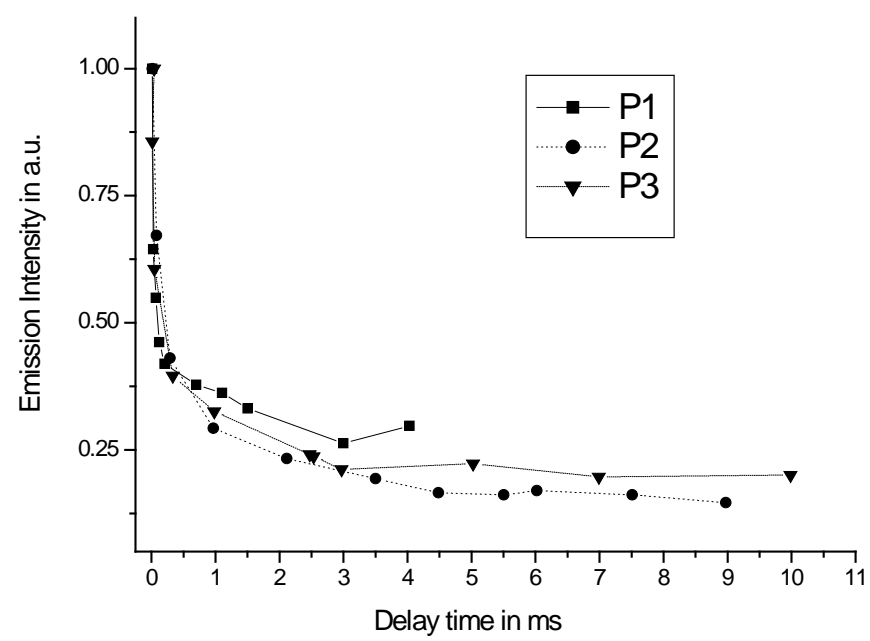

Fig. 5. Decay curves of P1, P2 and P3 (a) at low temperature (10 K) and (b) at RT, respectively. 
The energy gap between $\mathrm{S}_{1}$ and $\mathrm{T}_{1}$ states, $\Delta E_{\mathrm{ST}}, 0.61 \mathrm{eV}, 0.53 \mathrm{eV}$ and $0.52 \mathrm{eV}$ at $\mathrm{RT}$ for $\mathrm{P} 1, \mathrm{P} 2$ and $\mathrm{P} 3$ respectively.
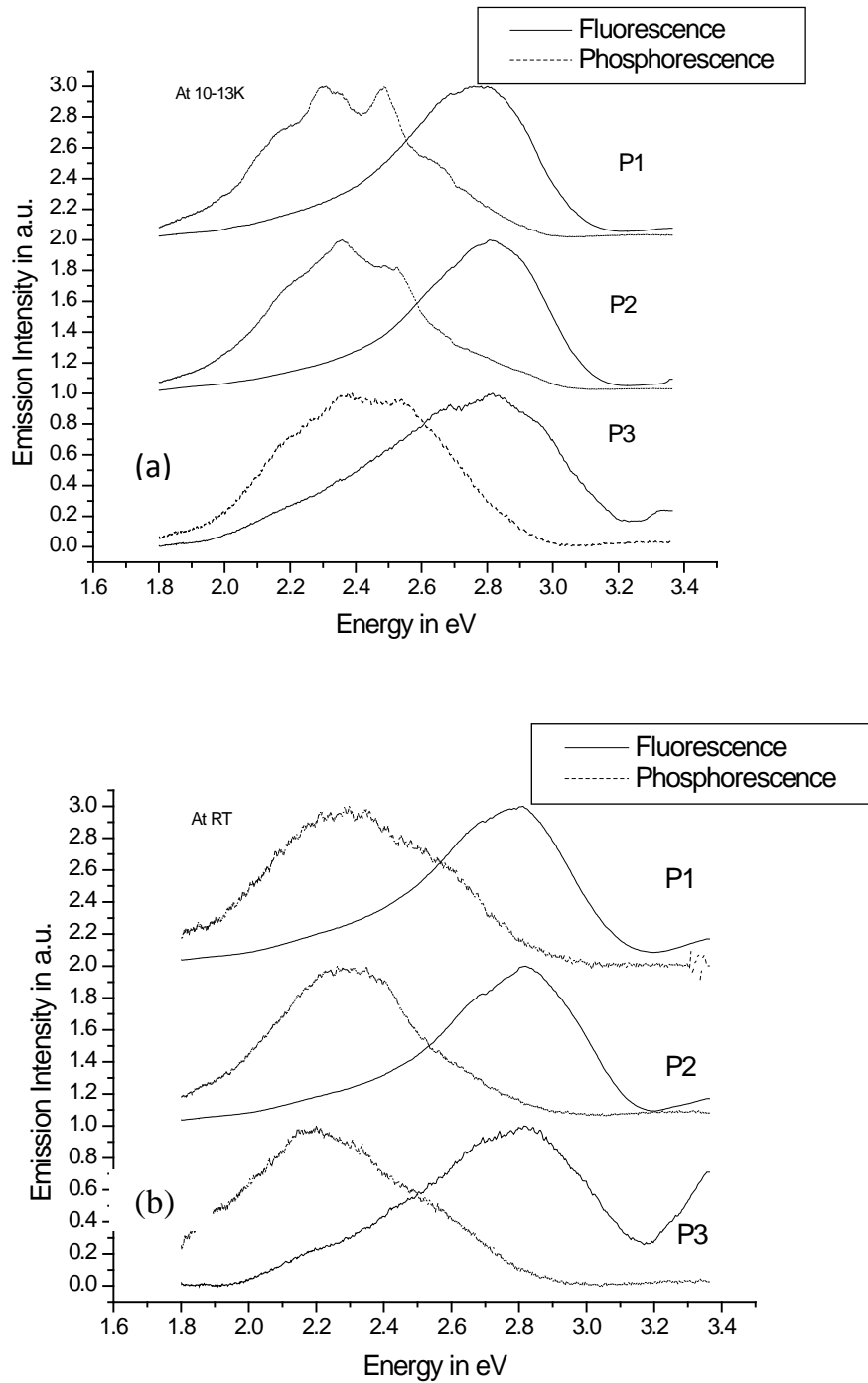

Fig. 6. Phosphorescence and Florescence (prompt) spectra (a) at low temperature (10 k) and (b) at RT, respectively.

In contrast to the fully allowed optical transitions for the singlet states, triplet state emission in conjugated polymers is at best only partially allowed and therefore has a long lifetime in the range of microseconds to seconds (see Fig. 4). For this reason the decay of triplet states is controlled by nonradiative mechanisms. These same nonradiative decay 
mechanisms also apply to the singlet states but are often insignificant in comparison to the fast radiative decay or intersystem crossing [19].

At room temperature, thermal broadening masks the vibronic structure. Diffusion of the longer-lived triplet excitations ( $45 \mu \mathrm{s}$ at $16 \mathrm{~K}$ for $(\mathrm{Pt}-\mathrm{TBT})_{\propto}$ ) [20] to quenching sites reduces the lifetime (to $2 \mu$ s at RT) [21] and thus the intensity of the triplet emission. In all of the investigated polymers, the life-time of triplet state is longer at low temperature than at RT.

The values for $\Delta E_{\mathrm{ST}}$ at low temperature (10-13K) and at RT for the three carbazolebased polymers lie in between the values found for Pt-polymers $\left(6 \pm 1 \mathrm{eV}^{-1}\right)$ and Ptmonomers $\left(3.8 \pm 0.5 \mathrm{eV}^{-1}\right)$ [20].

All decay curves at low temperature have been fitted mono-exponentially (see Fig. 5). The life-times were found $345 \mathrm{~ms}, 605 \mathrm{~ms}$ and $192 \mathrm{~ms}$ respectively for P1, P2 and P3 polymers at low temperature $(10-13 \mathrm{~K})$. However, fits to decay curves are multiexponential at RT (see Fig. 6). Therefore it may also consist of triplet-triplet annihilation which show fast decay times. All three materials show low energy tail in fluorescence spectra which may include emission from triplet excited state at both low and room temperature.

Table 1 summarizes steady-state, time-resolved and exponential fit to decay curves of P1, P2 and p3. For high-energy (green or blue) triplet emitters it is very important that triplet excitons should not be transferred from the triplet emitter to its host material. This requirement means that the triplet energy of the host should be higher than that of the triplet emitter. Frequently used polymers such as poly ( $p$-phenylenevinylene) (PPV) has a maximum triplet energy of 1.6, which limits the application as host polymers for triplet emitters to red or yellow triplet emitters. It is not straightforward to find a suitable host for high energy triplet emitters. Materials with a large HOMO-LUMO energy gap will automatically have high triplet energy. However, these materials will also suffer from charge injection problems due to misalignment of the HOMO and/or LUMO level with the Fermi levels of the electrodes (or charge injection layers).

A polymer has high triplet energy and at the same time suitable HOMO and LUMO levels for efficient charge injection. A polymer with a HOMO level at about $-5 \mathrm{eV}$ (as the work function of the hole injection layer, PEDOT: PSS, is $4.9 \mathrm{eV}$ ), a triplet energy of at least $2.5 \mathrm{eV}$, and a LUMO level at about $-2 \mathrm{eV}$ (to enable efficient injection of electrons from commonly used cathodes). For instance, for PPV the exchange energy is $0.7 \mathrm{eV}$ [22] The carbazole-oxadiazole polymers investigated here have the exchange energy below 0.5 $\mathrm{eV}$. These copolymers has a potential to use as a high energy triplet emitters in guest-host systems [23].

In Fig. 7, the highest-energy triplet vibronic subband of the polymer P3 is centered at $2.49 \mathrm{eV}$ which is taken as measure for the copolymer triplet energy. This triplet energy is significantly higher than that of poly (fluorene)s (2.2-2.4 eV) and poly $(p$ phenylenevinylene) is (1.3-1.6 eV) [24] the most commonly used polymers in PLEDs, and lower than that of PVK (3 eV). In fact, the triplet energy of the carbazole copolymer is a prominent host for triplet emitters in OLEDs. 
Table 1. Data from steady-state spectra, time-resolved spectra and exponential fit to decay curves for corresponding polymers.

\begin{tabular}{|c|c|c|c|c|}
\hline & Polymers & P1 & P2 & P3 \\
\hline \multicolumn{5}{|c|}{ Data from steady-state spectra } \\
\hline 1 & $\begin{array}{c}\mathrm{S}_{0} \rightarrow \mathrm{S}_{1}(\mathrm{eV}) \text { at } \mathrm{RT} \\
\quad \text { (Absorption) }\end{array}$ & 3.40 & 3.25 & 3.30 \\
\hline 2 & $\begin{array}{c}\mathrm{S}_{0} \rightarrow \mathrm{S}_{2}(\mathrm{eV}) \text { at } \mathrm{RT} \\
\quad \text { (Absorption) }\end{array}$ & 4.00 & 3.90 & 3.87 \\
\hline 3 & $\begin{array}{l}\mathrm{S}_{1} \rightarrow \mathrm{S}_{0}(\mathrm{eV}) \text { at } \mathrm{RT} \\
\text { (Fluromax/Emission) }\end{array}$ & 2.92 & 2.82 & 2.76 \\
\hline 4 & Stoke’s shift at RT (eV) & 0.48 & 0.43 & 0.54 \\
\hline \multicolumn{5}{|c|}{ Data from time-resolved spectra } \\
\hline 5 & $\mathrm{~S}_{1} \rightarrow \mathrm{S}_{0}(\mathrm{eV})$ at low tem. & 2.81 & 2.80 & 2.76 \\
\hline 6 & $\mathrm{~T}_{1} \rightarrow \mathrm{S}_{0}(\mathrm{eV})$ at low tem. & 2.37 & 2.35 & 2.29 \\
\hline 7 & $\Delta \mathrm{E}_{\mathrm{ST}}(\mathrm{eV})$ at low tem. & 0.44 & 0.45 & 0.47 \\
\hline 8 & $\mathrm{~S}_{1} \rightarrow \mathrm{S}_{0}(\mathrm{eV})$ at $\mathrm{RT}$ & 2.81 & 2.81 & 2.80 \\
\hline 9 & $\mathrm{~T}_{1} \rightarrow \mathrm{S}_{0}(\mathrm{eV})$ at $\mathrm{RT}$ & 2.20 & 2.28 & 2.28 \\
\hline 10 & $\Delta \mathrm{E}_{\mathrm{ST}}(\mathrm{eV})$ at $\mathrm{RT}$ & 0.61 & 0.53 & 0.52 \\
\hline \multicolumn{5}{|c|}{ Data from exponential fit to decay curves } \\
\hline 11 & Triplet Life-time at low tem. (ms) & 345 & 605 & 192 \\
\hline 12 & Triplet Life-time at RT (ms) & 1.13 & 2.50 & 0.97 \\
\hline 13 & Life-time of delayed FL (ms) & 0.03 & 0.20 & 0.02 \\
\hline
\end{tabular}

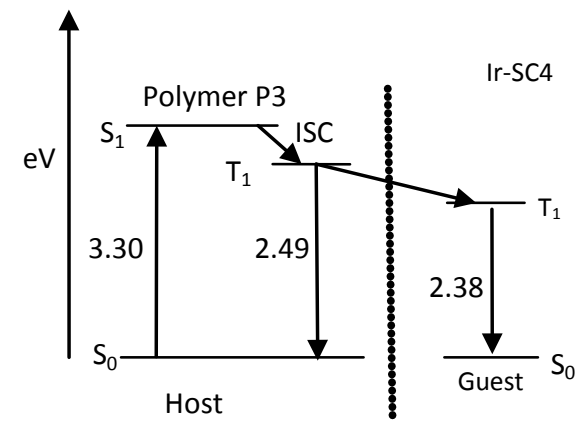

Fig. 7. The energy level diagram shows the energy levels of a host polymer and a triplet emitter. 


\section{Conclusion}

This series of mixed compounds enables us to study the influence of carbazole systems on the singlet and triplet energies. Furthermore, as carbazole derivatives are known to mainly transport holes, attaching electron transport moieties (such as oxadiazole derivatives) might be necessary to achieve balanced charge transport in OLEDs. Ultimately, this should lead to a design rule that results in compounds with high triplet energies and suitable HOMO and LUMO levels for charge injection by commonly used injection layers [17]. Carbazole based conjugated polymers are capable of emitting in the blue region of the spectrum. For the absorption process, among the three materials P2 has the lowest energy peaks because of alkyle group which delocalized carbazole system also the stabilizing effect is much stronger in these materials. Though they suffer from charge injection problem due to misalignment of HOMO and LUMO level further study regarding their internal structure, charge transport process and singlet-triplet mechanism will lead them to be the choice of material for manufacturing ideal OLED.

\section{Acknowledgments}

We gratefully owe to Jolanda Bastiaansen and Nicole. Kiggen for the synthesis of the three polymers that are used in this study.

\section{References}

1. N. Blouin, A. Michaud, D. Gendron, S. Wakim, E. Blair, R. Neagu-Plesu, M. Belletête, G. Durocher,Ye Tao, and M. Leclerc, J. Am. Chem. Soc. 130 (2), 732 (2008). doi:10.1021/ja0771989 ; PMid:18095689

2. N. Blouin and M. Leclerc, Acc. Chem. Res. 41 (9), 1110 (2008). doi:10.1021/ar800057k; PMid:18656967

3. T. Mori and M. Kijima, European Polymer Journal 45 (4), 1149, (2009). doi:10.1016/j.eurpolymj.2008.12.042

4. C. Rothe, S. King, and A. P. Monkman, Phys. Rev. B 73, 245208 (2006). doi:10.1103/PhysRevB.73.245208

5. J. V. Grazulevicius, P. Strohriegl, J. Pielichowski, and K. Pielichowski, Prog. Polym. Sci. 28, 1297 (2003). doi:10.1016/S0079-6700(03)00036-4

6. H. Yi, A. Iraqi, M. Stevenson, C. J. Elliott, and D. G. Lidzey, Macromol. Rapid Commun. 28, 1155 (2007). doi:10.1002/marc.200700076

7. T. Michinobu, H. Osako, and K. Shigehara, Micromol. Rapid Commun. 29, 111 (2008). doi:10.1002/marc. 200700715

8. N. J. Turro, Modern Molecular Photochemistry (University Science Books, Mill Valley, CA, 1991).

9. D. Beljonne, Z. Shuai, G. Pourtois, J. Bredas, J. Phys. Chem, 48 (9), 1249 (2009).

10. M. Belletete, J. Bouchard, M. Leclerc, and G. Durocher, Macromolecules 38, 880 (2005). doi:10.1021/ma048202t

11. N. Chawdhury, A. Kohler, R. H. Friend, W. Y. Wong, M. Younus, P. R. Raithby, P. R. Lewis, T. C. Corcoran, M. R. A. Mandhury, and M. S. Khan, J. Chem. Phys. 110, 4963 (1999). doi:10.1063/1.478382

12. S. Davies, J. Johnson, B. F. G. Khan, and M. S. Lewis, J. J. Chem. 23, 634 (1991).

13. R. H. Friend, D. D. C. Bradley, and P. D. Townsend, J. Phys. D: Appl. Phys. 20, 1367 (1987). 
14. S. Tokito, S. Saito, and R. Tanaka, Macromol. Chem. Rapid Common. 7, 557 (1986). doi:10.1002/marc.1986.030070904

15. J. L. Bredas, J. Cornil, and A. J. Heeger, Adv. Mater. 8, 447 (1996). doi:10.1002/adma.19960080517

16. T. W. Hagler, K. Packbaz, K. Voss, and A. J. Heeger, Phys. Rev. B 44, 8652 (1991). doi:10.1103/PhysRevB.44.8652

17. K. Brunner, A. van Dijken, H. Borner, J. J. A. M. Bastiaansen, N. M. M. Kiggen, and B. M. W. Langeveld, J. Am. Chem. Soc. 126, 6035 (2004). doi:10.1021/ja049883a ; PMid:15137768

18. J. F. Ambrose, L. L. Carpenter, R. F. Nelson, J. Electrochem. Soc.122, 876 (1975). doi:10.1149/1.2134365

19. J. S. Wilson, N. Chawdhury, M. R. A. Al-Mandhary, M. Younus, M. S. Khan, P. R. Raithby, A. Ko1hler, and R. H. Friend, J. Am. Chem. Soc. 123, 9412 (2001). doi:10.1021/ja010986s ; PMid:11562224

20. F. Wittmann, R. H. Friend, M. S. Khan, and J. Lewis, J. Chem. Phys. 101, 2693 (1994). doi:10.1063/1.467650

21. A. Kohler, F. Wittmann, R. H. Friend, M. S. Khan, and J. Lewis, Synthetic Metals 77, 147 (1996). doi:10.1016/0379-6779(96)80076-2

22. Y. Romanovskii, V. Gerhard, A. Schweitzer, B. Scherf, U. Personov, R. I. Ba“ssler, Phys. Rev. Lett. 84, 1027 (2000). doi:10.1103/PhysRevLett.84.1027 ; PMid:11017432

23. A. van Dijken, J. J. A. M. Bastiaansen, N. M. M. Kiggen, B. M. W. Langeveld, C. Rothe, A. Monkman, I. Bach, P. Sto"ssel, and K. Brunner, J. Am. Chem. Soc. 126, 7718 (2004). doi:10.1021/ja049771j; PMid:15198620

24. A. P. Monkman, P. Burrows, H. D. Hartwell, L. J. Horsburgh, L. E. Hamblett, and I. Navaratnam, Phys. Rev. Lett., 86, 1358. (2001). doi:10.1103/PhysRevLett.86.1358 PMid:11178083 